\title{
Outcomes of early inhaled nitric oxide use in premature African American neonates
}

\author{
Christopher A. Collura' ${ }^{1}$ Kristin C. Mara ${ }^{2} \cdot$ Amy L. Weaver ${ }^{2} \cdot$ Reese H. Clark ${ }^{3}$. William A. Carey ${ }^{1}$
}

Received: 2 May 2018 / Revised: 2 July 2018 / Accepted: 15 August 2018 / Published online: 1 October 2018

(c) Springer Nature America, Inc. 2018

\begin{abstract}
Objective Meta-analysis of individual-patient clinical trial data suggests that inhaled nitric oxide (iNO) improves respiratory outcomes in premature African American neonates. We hypothesized that early iNO therapy would be associated with lower mortality and less chronic lung disease (CLD) in extremely premature African American neonates.

Study design We conducted a retrospective cohort study of propensity score- and race-matched neonates 22-29 weeks gestation who were mechanically ventilated for treatment of respiratory distress and associated pulmonary hypertension $($ RDS + PPHN). We evaluated the association of iNO within 7 days of life with in-hospital mortality and CLD, using Cox proportional hazards regression and logistic regression, respectively.

Result Among 178 matched pairs of African American patients, iNO was not associated with lower mortality $(\mathrm{HR}=0.94$, 95\% CI 0.69-1.30) or less CLD (OR $=0.94,95 \%$ CI $0.47-1.87)$.

Conclusions Early, off-label iNO use is not associated with improved outcomes in premature African American neonates with RDS + PPHN.
\end{abstract}

\section{Introduction}

Inhaled nitric oxide (iNO) is not approved for use in neonates $<34$ weeks gestation as it neither improves survival nor prevents long-term morbidity in this population [1-14]. Both the American Academy of Pediatrics and the National Institutes of Health discourage its use in premature neonates, noting a lack of evidence to support iNO even among those with pulmonary hypertension (PPHN) $[15,16]$. In our recent study of extremely premature neonates with PPHN, we likewise found that the off-label prescription of

Electronic supplementary material The online version of this article (https://doi.org/10.1038/s41372-018-0232-6) contains supplementary material, which is available to authorized users.

William A. Carey

carey.william@mayo.edu

1 Division of Neonatal Medicine, Mayo Clinic, Rochester, MN, USA

2 Division of Biomedical Statistics and Informatics, Mayo Clinic, Rochester, MN, USA

3 Center for Research, Education \& Quality, Pediatrix Medical Group, Sunrise, FL, USA
iNO during the first week of life was not associated with improvement in survival or any of several major morbidities [17].

After completing the above cohort study, we were encouraged to learn that iNO may reduce the rate of chronic lung disease (CLD) in premature African American neonates [18]. Among the three clinical trials included in this individual-patient data meta-analysis [4, 10, 19], only one enrolled subjects exclusively during the first week of life [4]. This single-center study piqued our interest for two reasons: it revealed that iNO reduced the risk of death or CLD with a relatively low number needed to treat (seven), and a large majority of its subjects $(70 \%)$ were African American. The other two studies enrolled the majority of their subjects beyond the first week of life, with lesser treatment effects seeming to correlate with lower enrollment of African American subjects [10, 19].

Among 1278 neonates with PPHN in our recent study 382 (30\%) were African American [17], a proportion intermediate to those observed in two largest American trials of early iNO therapy $[6,9]$. Although neonates in our PPHN cohort were not matched directly on race, our posthoc assessment of African American neonates suggested that early initiation of iNO might improve outcomes in this population (unpublished data). We therefore conducted the 
present study, matching on race, to test our hypothesis that iNO use during the first week of life would be associated with lower mortality and less CLD among extremely premature African American neonates.

\section{Methods}

\section{Study design}

We performed a retrospective cohort study using data from the Clinical Data Warehouse (CDW). The CDW includes information on more than one million neonates who were hospitalized in a Pediatrix Medical Group (PMG) neonatal intensive care unit (NICU) [20]. The study was approved by the Western Institutional Review Board (IRB) and was considered exempt by the Mayo Clinic IRB (Rochester, MN).

\section{Study setting and population}

We identified all neonates admitted to a PMG NICU and discharged between 1 January 2004 and 31 December 2014. We included in our study singletons born at 22-29 weeks gestation who were mechanically ventilated for treatment of respiratory distress and concomitant PPHN. We excluded from our analysis neonates with major anomalies (including pulmonary hypoplasia), those admitted for comfort care only and those who died in the delivery room.

\section{Patient characteristics and outcomes}

We obtained various maternal and neonatal characteristics for each neonate in our study sample (Table 1). Race/ethnicity was based on maternal origin and was recorded in the electronic documentation tool as Asian, black (African American), Hispanic, Native American/Alaskan, Pacific Islander, white or other/unknown. The diagnosis of PPHN was at the discretion of the treating neonatologist-that is, there were no predetermined criteria required for diagnosis (e.g., echocardiography, pre-/post-ductal saturation differential, etc.). That said, in a prior study using this data set we demonstrated that PPHN was associated with increased mortality among patients with RDS [17], suggesting that this clinical diagnosis of PPHN is a meaningful discriminant. Information regarding iNO dosing and patients' responses to iNO was not available.

The primary outcome was in-hospital mortality, defined as death prior to transfer or discharge. Secondary outcomes included necrotizing enterocolitis of any stage (NEC); retinopathy of prematurity that required treatment (tROP); CLD, defined as a need for supplemental oxygen or pressure support at 36 weeks corrected gestational age (CGA); and periventricular leukomalacia (PVL).

\section{Data analysis}

Given our previous findings [17], we anticipated that patients who received iNO would have less-favorable baseline characteristics than those who did not receive iNO (i.e., they would seem "sicker"). We therefore used propensity score (PS) matching to balance the measured baseline characteristics between patients who received iNO during the first week of life and the referents to whom they were matched [21]. Because iNO could have been initiated at any time during the first week of life, we employed a risk set approach to match a patient who initiated iNO on day $t$ (exposed) to a patient with similar baseline characteristics who had not received iNO as of day $t$ (referent).

As previously described [17], we first built a risk set of all neonates "at risk" for receiving iNO on day $t=0$. Using these neonates, we fit a multivariable logistic model to estimate PS, defined as the probability that a neonate would receive iNO on day $t=0$ conditional on the measured covariates. This model included main effect terms for each of the variables listed in Table 1 along with race; gestational age, birth weight, and calendar year were modeled as linear terms, with an interaction between gestational age and inborn/outborn status. For each neonate who received iNO on day $t=0$, one matched referent was randomly selected without replacement from the pool of neonates who had not received iNO as of day $t=0$ that matched on race (African American, white, Hispanic, Asian, or other) and within \pm 0.2 standard deviations of the logit of the PS values. We then repeated this sequential process for each of the days $t=1-7$, excluding from each risk set neonates who had either received iNO, transferred, or died prior to time $t$. Last, we combined the matched pairs into race subgroups for analysis. The date of iNO initiation for an iNO-exposed patient was defined as the "index date" for both the iNOexposed and their matched referent.

We examined covariate imbalance between the exposed and referent groups by assessing the standardized difference for each baseline covariate. We defined the standardized difference for a continuous covariate as the absolute difference in group means divided by an estimate of the pooled standard deviation. The derivation is similar for nominal covariates. A standardized difference less than 0.10 signifies minimal covariate imbalance between groups [22].

The association between iNO exposure and each of the outcomes was evaluated separately for each race (African American, white, and Hispanic). We employed a Cox proportional hazards model to determine the association between iNO status and in-hospital mortality. We used age as the time scale, with neonates entering the risk set at their respective age at the index date [23]. Using the counting process formulation of a Cox model, neonates entered the analysis at their index age (left truncation) and exited at 


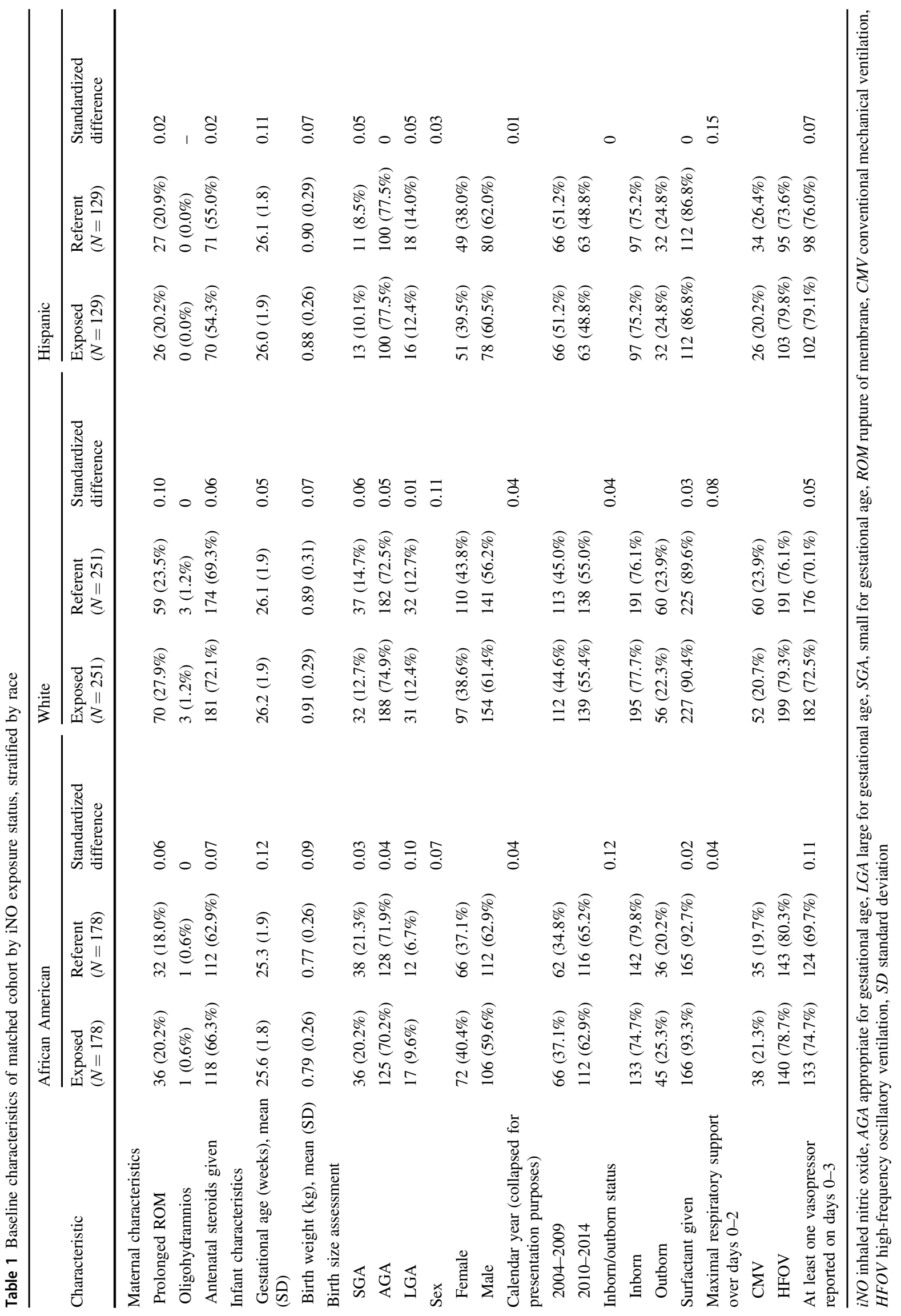


their death/transfer/discharge age. We used the robust sandwich covariance estimate in the Cox model to account for neonates included in both the exposed and referent cohorts (i.e., referents who later received iNO). Associations were summarized using the hazard ratio and corresponding $95 \%$ confidence interval (CI). We used the same time-to-event methodology to assess NEC as a secondary outcome. Last, we determined the duration of iNO therapy in each race sub-cohort by subtracting the age at which iNO was first initiated from either (a) the age at which it was discontinued or (b) the age at which a patient was transferred or died, if iNO was still prescribed that day.

For each of the other secondary outcomes we built separate PS- and race-matched sub-cohorts of patients who were eligible for evaluation of these diagnoses. For tROP we restricted the starting cohort to neonates who had been evaluated for ROP; for CLD we restricted the starting cohort to neonates still hospitalized at 36 weeks CGA; and for PVL we restricted the starting cohort to neonates who had undergone brain imaging. As iNO exposure status was known by the time each of these three outcomes was clinically evaluated, we estimated PS values and performed matching in one step for each sub-cohort. Thus, we considered iNO exposure status as a baseline covariate and evaluated the association between iNO and each secondary outcome using logistic regression. Associations were summarized using the odds ratio (OR) and corresponding 95\% confidence interval (CI). Unbalanced covariates in the PSmatched cohorts within each sub-cohort were adjusted for in the regression models evaluating the outcomes.

Last, we performed sensitivity analyses for the mortality and NEC outcomes to alternatively address the matched referents who subsequently received iNO within the first 7 days of life. Among the 558 referents in the PS- and racematched cohort, 147 received iNO within the first seven days of life, of whom only 124 also had been included in the matched cohort as "exposed" as there were no matched referents for the remaining 23 based on our matching calipers. In the sensitivity analysis using all 558 PS-matched pairs, we censored the follow-up of these 147 referents at the age they received iNO rather than at the age of their death/transfer/discharge. In this manner, the 124 matched referents who were also in the exposed group were handled as "referents" in the time interval prior to receiving iNO and as "exposed" in the time interval after receiving iNO.

\section{Results}

There were 73,594 neonates born at 22-29 weeks gestation and admitted to PMG NICUs from 2004 to 2014, during which time iNO was prescribed to at least $1 \%$ of neonates in this gestational age range. As shown in Fig. 1, a total of

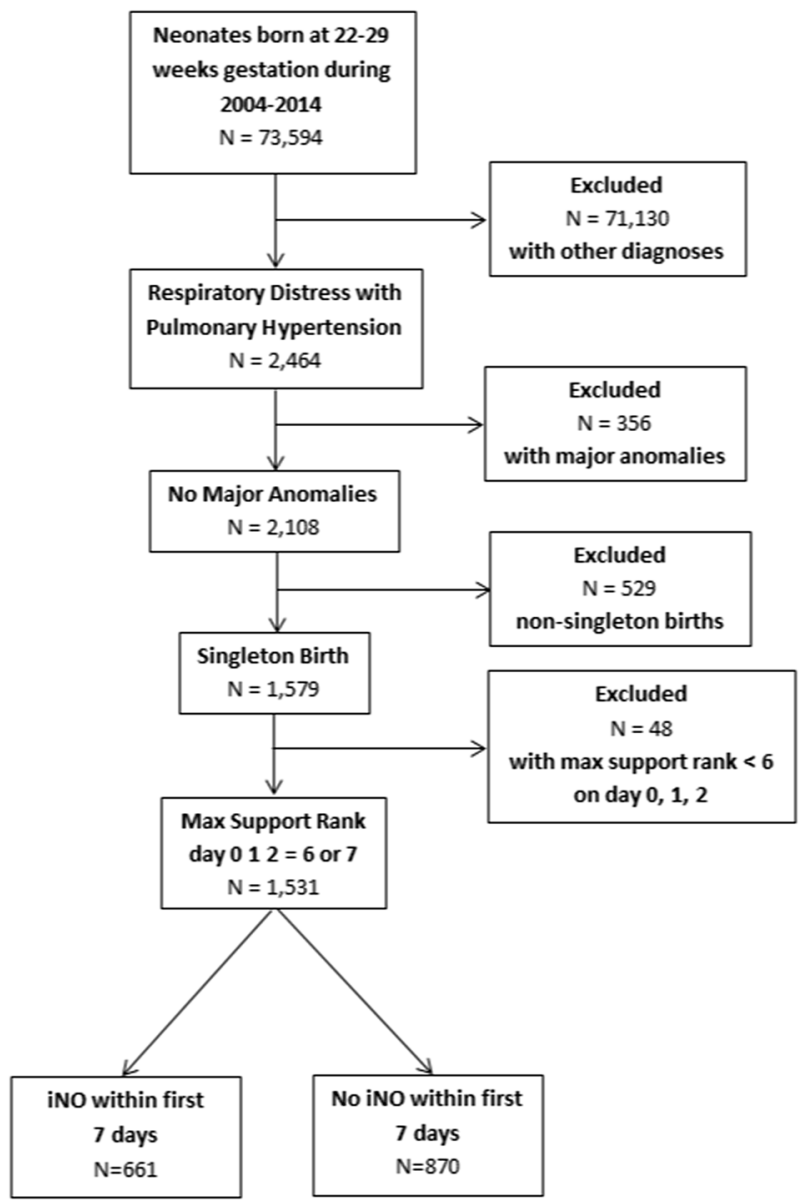

Fig. 1 Consort flow diagram

2464 patients received a primary respiratory diagnosis of RDS + PPHN. From this group of patients we sequentially excluded those with major congenital anomalies; nonsingleton birth status; or no requirement for either conventional or high-frequency ventilation on days $0-2$ ( $\max$ support rank 6 or 7 , respectively).

The analysis cohort was comprised of 1531 patients, of whom $661(43.2 \%)$ initiated iNO therapy within the first 7 days of life. The majority of iNO therapy was initiated soon after birth: $313(47.4 \%)$ initiated on day 0, 172 (26.0\%) initiated on day 1, $68(10.3 \%)$ initiated on day 2 and $108(16.3 \%)$ initiated on days 3-7. Characteristics of this unmatched cohort can be found in Supplemental Table 1. Most notably, patients treated with iNO more often required high-frequency ventilation and vasopressor therapy.

To reduce the imbalance in observed baseline covariates between iNO-exposed and referent patients, we built a cohort matched on race and PS values derived from all of the characteristics listed in Table 1. As shown by the standardized differences in Table 1 and Supplemental Fig. 1, there was fairly good covariate balance within each 
A

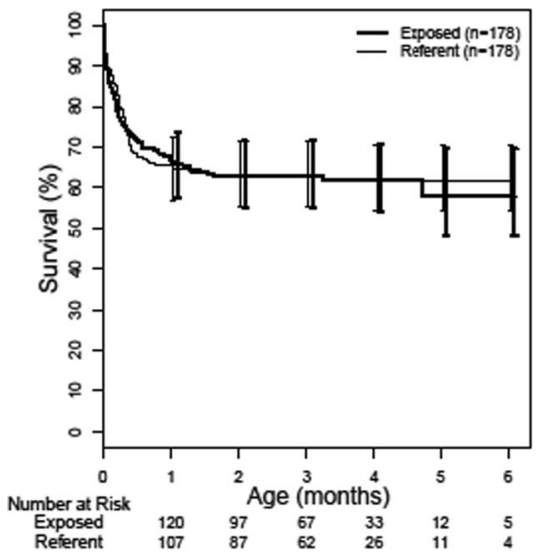

B

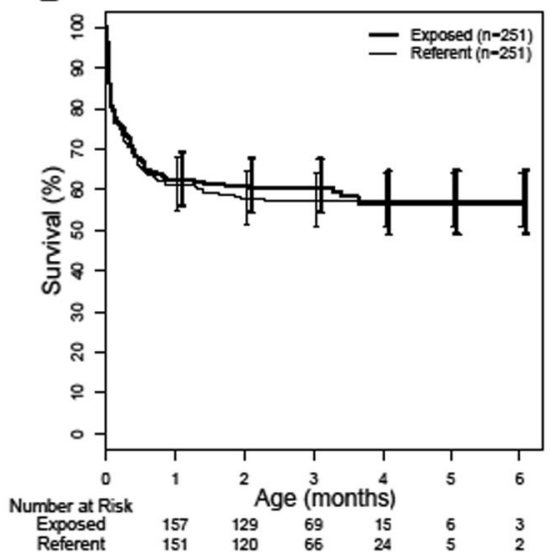

C

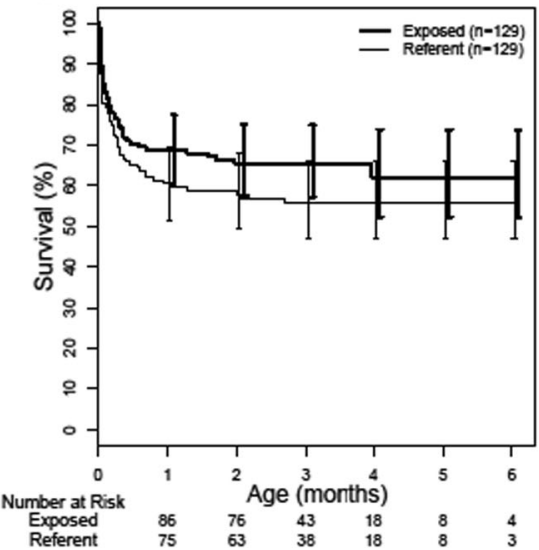

Fig. 2 Overall survival by iNO exposure status of a African American patients in the matched cohort, $\mathbf{b}$ white patients in the matched cohort, c Hispanic patients in the matched cohort
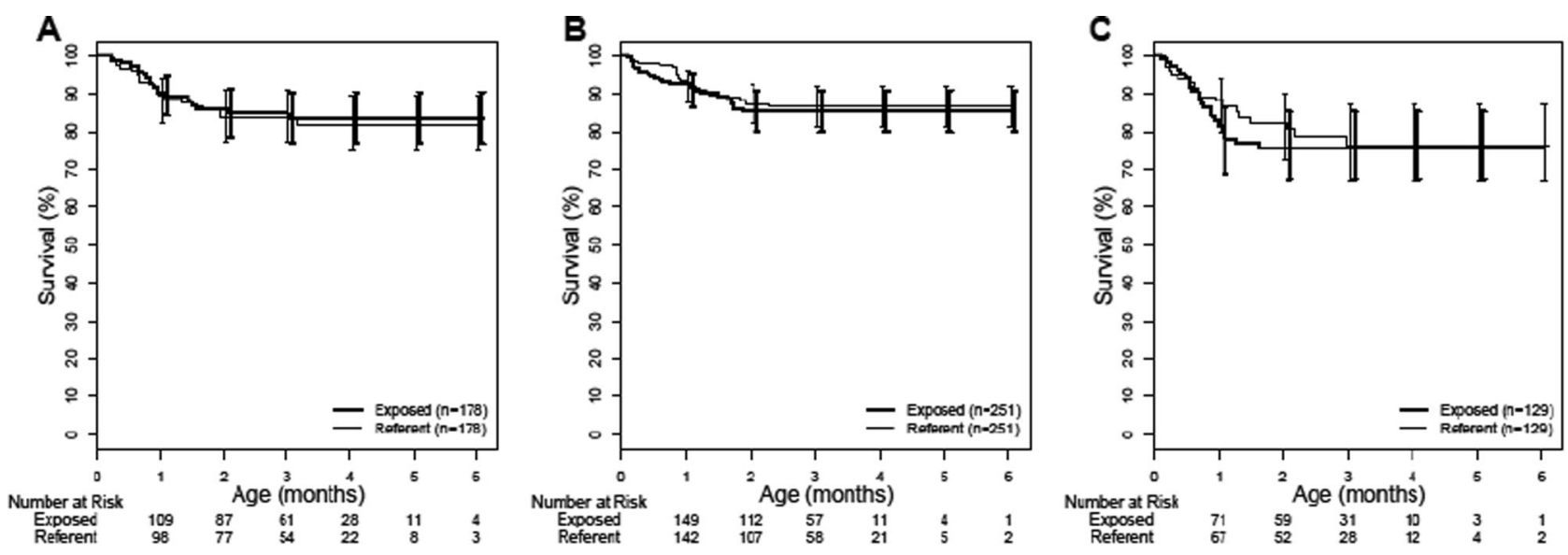

Fig. 3 NEC free survival by iNO exposure status of a African American patients in the matched cohort, $\mathbf{b}$ white patients in the matched cohort, c Hispanic patients in the matched cohort

of race sub-cohorts (African American, $n=356$; white, $n=502$; Hispanic, $n=258$ ). The median duration of iNO therapy was 5 days for all sub-cohorts (African American: IQR, 3-9 days; white: IQR, 4-7 days; Hispanic: IQR, 3-8 days).

The primary outcome was death prior to transfer/discharge, and neonates were followed until the date of their discharge/transfer. Among the neonates in the iNO-exposed group, 58 African Americans died at a median age of nine days (IQR, 4-25 days); 85 whites died at a median age of seven days (IQR, 2-14 days); and 39 Hispanics died at a median age of 5 days (IQR, 2-11 days). Among the matched referents, 58 African Americans died at a median age of nine days (IQR, 5-13 days); 91 whites died at a median age of six days (IQR, 2-14 days); and 48 Hispanics died at a median age of 7 days (IQR, 2-13 days). As shown in Fig. 2, we did not observe a significant association between iNO exposure and in-hospital mortality within any of the race sub-cohorts (African Americans: $\mathrm{HR}=0.94,95 \%$
CI: $0.69-1.30$; whites: $\mathrm{HR}=0.92,95 \%$ CI: $0.71-1.20$; Hispanics, adjusted for max support rating: $H R=0.74$, 95\% CI 0.51-1.08).

Among the neonates in the iNO-exposed group, 20 African Americans were diagnosed with NEC at a median age of 27 days (IQR, 19-43 days); 25 whites were diagnosed at a median age of 20 days (IQR, 8-46 days); and 22 Hispanics were diagnosed at a median age of 22 days (IQR, 16-30 days). Among the matched referents, 20 African Americans were diagnosed with NEC at a median age of 27 days (IQR, 1-42 days); 21 whites were diagnosed at a median age of 28 days (IQR, 24-40 days); and 18 Hispanics were diagnosed at a median age of 21 days (IQR, 9-40 days). Our analyses did not reveal a significant association between iNO exposure and NEC within any of the race subcohorts (African Americans: HR $=0.88,95 \%$ CI: 0.52 1.48; whites: $\mathrm{HR}=1.23$, 95\% CI: 0.75-2.03; Hispanics, adjusted for max support rating: $\mathrm{HR}=1.09,95 \%$ CI $0.61-$ 1.97; Fig. 3). Among the remaining secondary outcomes, 
early iNO exposure was associated only with a lower rate of CLD in Hispanics (adjusted for birth size assessment and inborn/outborn status, OR $=0.39,95 \% \mathrm{CI}$ : $0.17-0.87)$ and a higher rate of PVL in African Americans $(\mathrm{OR}=2.45$, 95\% CI: 1.03-5.85).

The results from the sensitivity analysis were consistent with the primary analysis. There was no association between iNO exposure and in-hospital mortality within any of the race sub-cohorts (African Americans: $\mathrm{HR}=1.08$, 95\% CI: 0.72-1.64; whites: $\mathrm{HR}=1.11,95 \%$ CI: 0.80-1.55; Hispanics, adjusted for $\max$ support rating: $H R=0.81$, 95\% CI 0.51-1.28). Likewise, there was no association between iNO exposure and NEC (African Americans: HR $=0.87$, 95\% CI: 0.4-1.70; whites: $\mathrm{HR}=1.22$, 95\% CI: 0.65-2.32; Hispanics, adjusted for max support rating: HR $=1.06,95 \%$ CI $0.55-2.03$ ).

\section{Discussion}

After two decades of conflicting trial data and much debate in the field of neonatology, it was encouraging to read that a subgroup of premature neonates might truly benefit from iNO therapy [18]. Compared with white and Hispanic neonates, non-Hispanic black neonates endure higher rates of prematurity, low birth weight and death at $<28$ days of life [24, 25]. Disparities in care delivery are known to exacerbate these problems [26, 27]. Thus, it was hopeful to think that iNO might represent a new means by which to close the long-standing quality gap experienced by African American neonates.

Unfortunately, among a large cohort of patients with RDS + PPHN we found that early iNO treatment was not associated with less CLD in African Americans. This outcome is discordant with that of the RiNOP study [18], a meta-analysis in which at least one-third of the African American subjects were enrolled during the first week of life. Compared with the trial conducted by Schreiber et al. [4], mortality was higher among patients in our PS-matched cohort and CLD was more common among survivors. These outcomes disparities may relate to the fact that our patients were smaller, less mature and had more severe baseline characteristics (similar to patients enrolled in the PiNO trial) [6]. However, since the time of the Schreiber publication most iNO-treated neonates $<30$ weeks gestation have RDS that is accompanied by a diagnosis of PPHN [17]. There also is evidence that early iNO therapy may be harmful when it is prescribed to treat RDS in the absence of PPHN [17]. Thus, it is still unclear whether there truly is a subpopulation of premature neonates who might benefit from iNO.

Similarly, the optimal dosage and timing of iNO was not clarified by the RiNOP meta-analysis. For example,
Schreiber et al. initiated iNO at $10 \mathrm{ppm}$ for 1 day, then weaned to 5 ppm 6 days [4]. The NEWNO and NO CLD trials initiated iNO at $20 \mathrm{ppm}$ for up to 4 days, continued at $10 \mathrm{ppm}$ for 1 week, then weaned further to complete at least 24 total days of therapy [10, 19]. Likewise, Schreiber et al. enrolled most of their subjects at $<1$ day of life [4], whereas the NEWNO and NO CLD trials enrolled the majority of their subjects during the second and third week of life, respectively [10, 19]. Age at enrollment is important to consider because it influences the outcomes of neonatal study populations, survival in particular [27]. Most mortality in the NICU occurs during the first week of life. Because this phenomenon is inversely proportional to gestational age [27], the potential for survivorship bias prevents us drawing any conclusions from the RiNOP study. In our present study, African American neonates initiated iNO at a median age of one day and received just 5 days of treatment (IQR 3-9 days). Although our patients' comparably brief (and possibly lower-dose) iNO course could explain the null result, it is worth noting that the NO CLD and NEWNO trials yielded divergent outcomes despite their similar study populations and iNO administration protocols [10, 19].

In addition to the unexpectedly null result, we identified [28] two outcomes of early iNO exposure that are worth mentioning. First, exposure to iNO was associated with a much lower rate of CLD among Hispanic neonates (Table 2). One-quarter of the 220 matched pairs whom we assessed for CLD were Hispanic, a proportion that is higher than that of the RiNOP study $(\approx 15 \%$ ) but nearly identical to the percentage of American live births $<32$ weeks gestation [24]. Because Hispanic ethnicity appears to be protective against RDS and PPHN [29, 30], it is conceivable that Hispanic patients might respond more favorably to iNO therapy than neonates of other racial backgrounds. Second, we were troubled to find an association between early iNO exposure and PVL in the African American sub-cohort (Table 2). This finding might be spurious, as the relatively small sample size of each race sub-cohort prevented us from matching on potential confounders, such as center and gestational age. Nevertheless, out of caution we conclude that iNO should not be prescribed for the prevention of bronchopulmonary dysplasia in this subpopulation. Instead, and bearing in mind the limitations of the present study and the RiNOP meta-analysis, we strongly endorse the conduct of clinical trials that specifically enroll African American and Hispanic neonates. Even in the era of the 21st Century Cures Act, adequately powered and generalizable clinical trials remain the gold standard in evidence-based medicine. Based on our review of the literature [6, 17, 18, 24], we suggest that a trial of early iNO in extremely premature African American and Hispanic neonates with PPHN would be an important and feasible step toward eliminating the 


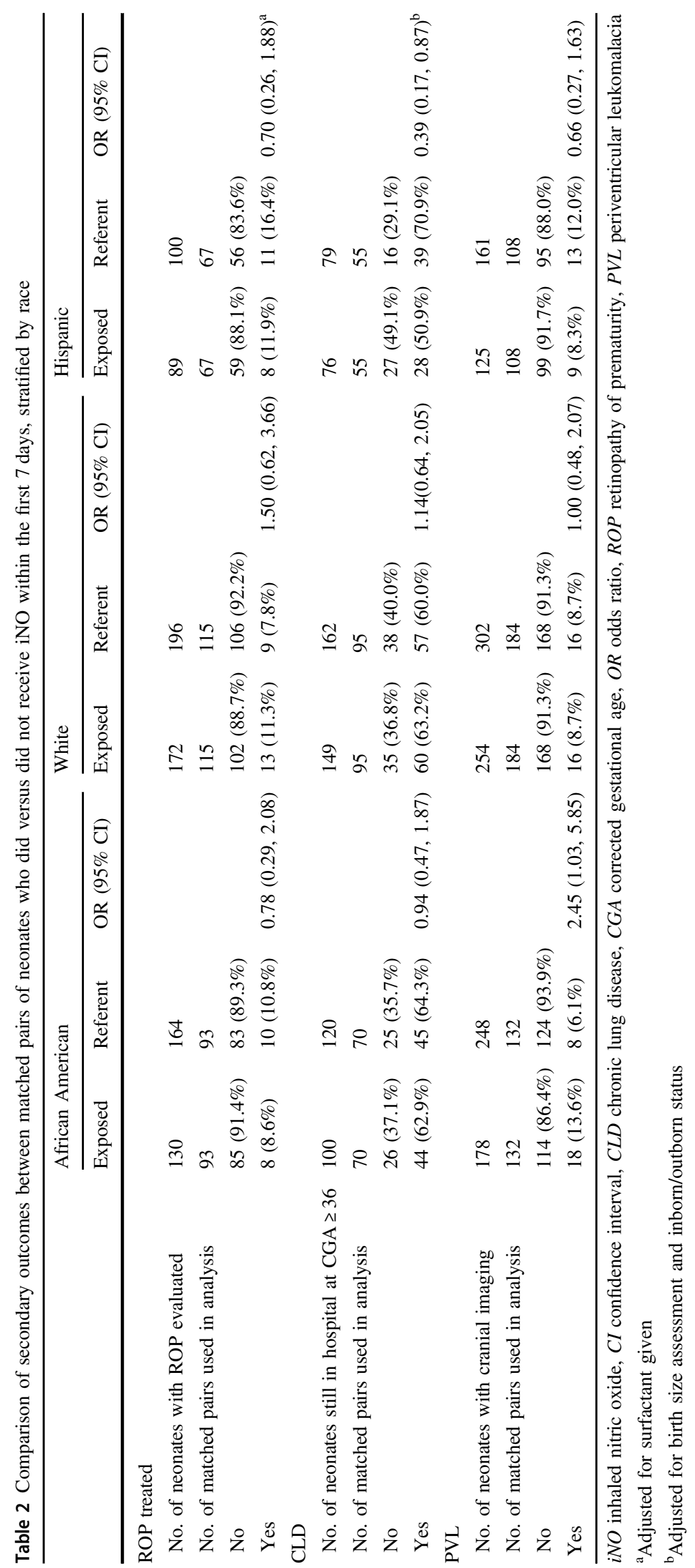


disparities that currently plague our system on neonatal care $[26,27]$.

\section{Limitations and strengths}

Our retrospective study design posed a few limitations that we address here. As we state above, our patients' diagnosis of PPHN was made at the discretion of the treating neonatologists. Likewise, we were unable to determine the doses at which PMG neonatologists initiated, escalated or weaned iNO in response to their patients' clinical status. As a result, we are unable to compare the outcomes of this study to those included in the RiNOP study [4, 10, 19]. Next, in our PS model we included only one variable that directly reflected the severity of our patients' respiratory disease: maximum level of ventilator support on days of life 0-2. Although it would have been valuable to include other characteristics (e.g., oxygenation index), we were limited to those that were included in our data source. Last, our study design also impacted our assessment of secondary outcomes. For example, we could not assess intraventricular hemorrhage (IVH) because we could not determine with certainty whether a patient's IVH occurred before or after initiating iNO. For the three secondary outcomes we did assess, the number of patients included in each analysis was limited by our need to PS- and race-match patients who were alive and eligible for evaluation of these diagnoses. For the same reason, we were unable to match patients on site-for example, we compared the survival of 178 PSmatched pairs of African American patients and there are $>$ 300 hospitals included in our data source (PMG CDW).

Perhaps the greatest strength of this study is quality of the CDW data. As reported by our group and others, the rate of off-label iNO utilization by PMG neonatologists is similar to those of the New South Wales/Australian Capital Territory network and the Vermont-Oxford Network [20, 31]. Furthermore, the demographic characteristics and clinical outcomes of our PS-matched cohort were comparable to those of the two largest multicenter trials of early iNO conducted in the United States [6, 9]. Thus the PMG experience-its physicians' practices and its patients' response those practices-seems generalizable to the broader neonatology community.

\section{Conclusion}

Off-label prescription of iNO does not improve respiratory outcomes in extremely premature African American neonates with RDS + PPHN. Along with recent meta-analyzed clinical trial data, our findings provide additional evidence that a clinical trial is warranted in this at-risk population.

\section{Compliance with ethical standards}

Funding Data analysis was funded by the Mayo Clinic Children's Research Center.

Conflict of interest Reese H. Clark reports support for CME conferences from Mallinckrodt Pharmaceuticals. The remaining authors declare no conflicts of interest.

\section{References}

1. Subhedar NV, Shaw NJ. Changes in oxygenation and pulmonary haemodynamics in preterm infants treated with inhaled nitric oxide. Arch Dis Child Fetal Neonatal Ed. 1997;77:F191-97.

2. Kinsella JP, Walsh WF, Bose CL, Gerstmann DR, Labella JJ, Sardesai $\mathrm{S}$, et al. Inhaled nitric oxide in premature neonates with severe hypoxaemic respiratory failure:a randomised controlled trial. Lancet. 1999;354:1061-5.

3. Srisuparp P, Heitschmidt M, Schreiber MD. Inhaled nitric oxide therapy in premature infants with mild to moderate respiratory distress syndrome. J Med Assoc Thai. 2002;85:S469-78.

4. Schreiber MD, Gin-Mestan K, Marks JD, Huo D, Lee G, Srisuparp P. Inhaled nitric oxide in premature infants with the respiratory distress syndrome. N Engl J Med. 2003;349:2099107.

5. Field D, Elbourne D, Truesdale A, Grieve R, Hardy P, Fenton AC, et al. Neonatal ventilation with inhaled nitric oxide versus ventilatory support without inhaled nitric oxide for preterm infants with severe respiratory failure: the INNOVO multicentre randomized controlled trial. Pediatrics. 2005;115:926-36.

6. Van Meurs KP, Wright LL, Ehrenkranz RA, Lemons JA, Ball $\mathrm{MB}$, Poole WK, et al. Inhaled nitric oxide for premature infants with severe respiratory failure. N Engl J Med. 2005;353:13-22.

7. Hascoet JM, Fresson J, Claris O, Hamon I, Lombet J, Liska A, et al. The safety and efficacy of nitric oxide therapy in premature infants. J Pediatr. 2005;146:318-23.

8. Dani C, Bertini G, Pezzati M, Filippi L, Cecchi A, Rubaltelli FF. Inhaled nitric oxide in very preterm infants with severe respiratory distress syndrome. Acta Paediatr. 2006;95:1116-23.

9. Kinsella JP, Cutter GR, Walsh WF, Gerstmann DR, Bose CL, Hart C, et al. Early inhaled nitric oxide therapy in premature newborns with respiratory failure. $\mathrm{N}$ Engl J Med. 2006; 355:354-64.

10. Ballard RA, Truog WE, Cnaan A, Martin RJ, Ballard PL, Merrill $\mathrm{JD}$, et al. Inhaled nitric oxide in preterm infants undergoing mechanical ventilation. N Engl J Med. 2006;355:343-53.

11. Mercier JC, Hummler H, Durrmeyer X, Sanchez-Luna M, Carnielli $\mathrm{V}$, Field $\mathrm{D}$, et al. Inhaled nitric oxide for prevention of bronchopulmonary dysplasia in premature babies(EUNO): a randomised controlled trial. Lancet. 2010;376:346-54.

12. Barrington KJ, Finer N, Pennaforte T. Inhaled nitric oxide for respiratory failure in preterm infants. Cochrane Database Syst Rev. 2010;CD000509.

13. Donohue PK, Gilmore MM, Cristofalo E, Wilson RF, Weiner JZ, Lau BD, et al. Inhaled nitric oxide in preterm infants: a systematic review. Pediatrics. 2011;127:e414-22.

14. Askie LM, Ballard RA, Cutter GR, Dani C, Elbourne D, Field D, et al. Inhaled nitric oxide in preterm infants: an individualpatient data meta-analysis of randomized trials. Pediatrics. 2011;128:729-39.

15. Kumar P. Committee on fetus and newborn, American Academy of pediatrics. Use of inhaled nitric oxide in preterm infants. Pediatrics. 2014;133:164-70. 
16. Cole FS, Alleyne C, Barks JD, Boyle RJ, Carroll JL, Dokken D, et al. NIH consensus development conference statement: inhaled nitric-oxide therapy for premature infants. Pediatrics. 2011; 127:363-9.

17. Carey WA, Weaver AL, Mara KC, Clark RH. Inhaled nitric oxide in extremely premature neonates with respiratory distress syndrome. Pediatrics. 2018;141:e20173108.

18. Askie LM, Davies LC, Schreiber MD, Hibbs AM, Ballard PL, Ballard RA. Race effects of inhaled nitric oxide in preterm infants: an individual participant data meta-analysis. J Pediatr. 2018; 193:34-39.

19. Hasan SU, Potenziano J, Konduri GG, Perez JA, Van Meurs KP, Walker MW, et al. Effect of inhaled nitric oxide on survival without bronchopulmonary dysplasia in preterm infants: a randomized clinical trial. JAMA Pediatr. 2017;171: 1081-9.

20. Ellsworth MA, Harris MN, Carey WA, Spitzer AR, Clark RH. Off-label use of inhaled nitric oxide after release of NIH consensus statement. Pediatrics. 2015;135:643-8.

21. D'Agostino RB Jr. Propensity score methods for bias reduction in the comparison of a treatment to a non-randomized control group. Stat Med. 1998;17:2265-81.

22. Austin PC. Balance diagnostics for comparing the distribution of baseline covariates between treatment groups in propensity-score matched samples. Stat Med. 2009;28:3083-107.
23. Therneau TM, Grambsch PM. Modeling survival data: extending the Cox model. New York, NY: Spriger-Verlag; 2000.

24. Martin JA, Hamilton BE, Osterman MJK, Driscoll AK, Drake P. Births: final data for 2016. Natl Vital Stat Rep. 2018;67:1-55.

25. Murphy SL, Xu J, Kochanek KD, Curtin SC, Arias E. Deaths: final data for 2015. Natl Vital Stat Rep. 2018;66:1-75.

26. Profit J, Gould JB, Bennett M, Goldsetin BA, Draper D, Phibbs CS, et al. Racial/ethnic disparity in NICU quality of care. Pediatrics. 2017;172:17-23.

27. Howell EA, Janevic T, Hebert PL, Egorova NN, Balbierz A, Zeitlin J. Differences in morbidity and mortality rates in black, white and Hispanic very preterm infants among New York hospitals. JAMA Pediatr. 2018;172:269-77.

28. Hornik CP, Sherwood AL, Cotten CM, Laughon MM, Clark RH, Smith PB. Daily mortality of infants born at less than 30weeks' gestation. Early Hum Dev. 2016;99:27-30.

29. Steurer MA, Jelliffe-Pawlowski LL, Baer RJ, Partridge JC, Rogers EE, Keller RL. Persistent pulmonary hypertension of the newborn in late preterm and term infants in California. Pediatrics. 2017;139:e20161165.

30. Wallace ME, Mendola P, Kim SS, Epps N, Chen Z, Smarr M, et al. Racial/ethnic differences in preterm perinatal outcomes. Am J Obstet Gynecol. 2017;216:306.e1-12.

31. Finer NN, Evans N. Inhaled nitric oxide for the preterm infant: evidence versus practice. Pediatrics. 2015;135:754-6. 